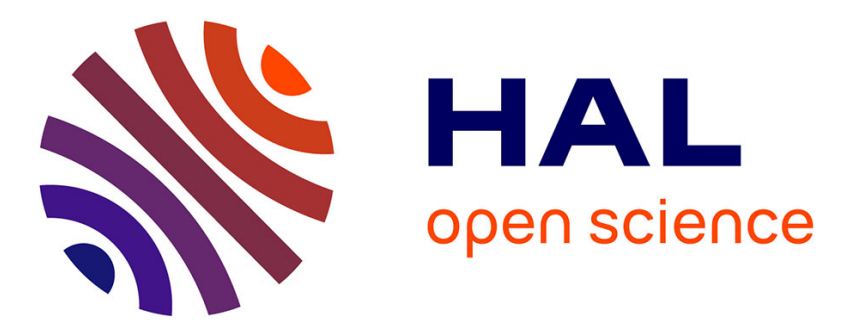

\title{
Comparison and experimental validation of two potential resonant viscosity sensors in the kilohertz range
}

\author{
Etienne Lemaire, Martin Heinisch, Benjamin Caillard, Bernhard Jakoby, \\ Isabelle Dufour
}

\section{- To cite this version:}

Etienne Lemaire, Martin Heinisch, Benjamin Caillard, Bernhard Jakoby, Isabelle Dufour. Comparison and experimental validation of two potential resonant viscosity sensors in the kilohertz range. Measurement Science and Technology, 2013, 24, 084005 (10 p.). 10.1088/0957-0233/24/8/084005 . hal-00843397

\section{HAL Id: hal-00843397 \\ https://hal.science/hal-00843397}

Submitted on 13 Nov 2014

HAL is a multi-disciplinary open access archive for the deposit and dissemination of scientific research documents, whether they are published or not. The documents may come from teaching and research institutions in France or abroad, or from public or private research centers.
L'archive ouverte pluridisciplinaire HAL, est destinée au dépôt et à la diffusion de documents scientifiques de niveau recherche, publiés ou non, émanant des établissements d'enseignement et de recherche français ou étrangers, des laboratoires publics ou privés. 


\title{
Comparison and experimental validation of two potential resonant viscosity sensors in the kilohertz range
}

\author{
Etienne Lemaire $^{1}$, Martin Heinisch ${ }^{2}$, Benjamin Caillard ${ }^{1}$, Bernhard Jakoby ${ }^{2}$, \\ Isabelle Dufour ${ }^{1}$ \\ ${ }^{1}$ Univ. Bordeaux, IMS, UMR 5218, F-33400 Talence, France \\ ${ }^{2}$ Institute for Microelectronics and Microsensors, Johannes Kepler University, Linz, \\ Austria \\ E-mail: etienne.lemaire@u-bordeaux1.fr
}

\begin{abstract}
Oscillating microstructures are well-established and find application in many fields. These include, force sensors, e.g. AFM micro-cantilevers or accelerometers based on resonant suspended plates. This contribution presents two vibrating mechanical structures acting as force sensors in liquid media in order to measure hydrodynamic interactions. Rectangular cross section microcantilevers as well as circular cross section wires are investigated. Each structure features specific benefits, which are discussed in detail. Furthermore, their mechanical parameters and their deflection in liquids are characterized. Finally, an inverse analytical model is applied to calculate the complex viscosity near the resonant frequency for both types of structures. With this approach it is possible to determine rheological parameters in the kilo Hertz range in situ within a few seconds. The monitoring of the complex viscosity of yogurt during the fermentation process is used as a proof of concept to qualify at least one of the two sensors into opaque mixtures.
\end{abstract}

\section{Introduction}

Vibrating microstructures are known to interact with the surrounding medium they are immersed in $[1,2]$. The dynamic mechanical motion of such structures is used to sense the parameters of interest. The aim of the study is to compare two types of structures for a viscoelasticity sensing applications. The vibrating wires and microcantilevers described in this paper are electromagnetically actuated, which is compatible with most liquids. Vibration measurements are obtained electrically by a piezoresistive Wheatstone bridge set-up in the case of the microcantilever [3] and by measuring the motion induced voltage in the case of the wire [4]. These methods can be efficiently used to characterize the mechanical parameters in the vicinity of the resonant frequency. In order to cover a larger spectral range containing all modes of vibration, an optical measurement is also performed using a laser Doppler vibrometer (MSA-500 from Polytec).

Complex viscous fluids feature frequency-dependent properties, e.g., viscoelastic phenomena can occur at specific shear rates or frequencies (in case of oscillating motions). In general, resonant microstructures can be used to measure hydrodynamic forces which depend on both, the fluid density and the complex viscosity [5]. An analytical model, some parts of which are detailed in this paper, allows characterizing the complex fluids by recording the frequency dependent deflections of these microstructures being immersed in liquids. In order to obtain reliable results, a well-controlled experimental set-up as well as a complete physical characterization of the microstructures was performed.

Transparent fluids (e.g., water or egg albumin) have been characterized using an optical characterization, whereas opaque fluids (e.g., yogurt) have been measured using an electrical measurement. The comparison between the results obtained with both structures allows for verifying the validity of the developed analytical model. The monitoring of the complex viscosity of yogurt during the fermentation process has been done in order to compare and validate the obtained rheological results with data from literature [6, 7]. 


\section{Materials and Methods}

\subsection{Description of the mechanical structures}

The microcantilevers are designed and manufactured in collaboration with the LAAS in Toulouse (www.laas.fr.). The process uses SOI wafers enabling controlling the microcantilever's thickness (using the buried layer of silicon dioxide as etch-stop layer). Thus, the thickness is fixed between 19.7 and $20.3 \mu \mathrm{m}$. Three cantilevers with different geometries (see Table I) were designed, and are denoted as $\mathrm{A}, \mathrm{LL}$ and $\mathrm{LH}$.

Table 1. Dimensions of the fabricated microcantilevers.

\begin{tabular}{llll} 
Cantilevers & A & LL & LH \\
\hline Length $(\boldsymbol{\mu m})$ & 500 & 2810 & 1440 \\
Width $(\boldsymbol{\mu m})$ & 100 & 100 & 285 \\
Thickness $(\boldsymbol{\mu m})$ & 20 & 20 & 20 \\
\hline
\end{tabular}

The cantilever's mass density is $2.4510^{3} \mathrm{~kg} \cdot \mathrm{m}^{-3}$, (silicon, the main constituent of the cantilever, has a density of $\left.2.3310^{3} \mathrm{~kg} \cdot \mathrm{m}^{-3}\right)$. The conducting paths, deposited on the structure are constituted of titanium $(100 \mathrm{~nm}$ thick) and then gold $(700 \mathrm{~nm})$. An insulating layer of silicone dioxide, $600 \mathrm{~nm}$ thick, is deposited at the end of the process on the cantilever covering the metal. The Young modulus for the complete structure is found to be about $150 \mathrm{GPa}$. The cantilever design includes a piezoresistive strain gauge overlapping the cantilever's clamping region. A pair of the fabricated cantilevers is shown in figure 1. The cantilever on the right is released, allowing for out of plane oscillations. The second one on the left is more than ten times thicker and thus static. It is subjected to the same physical and electrical conditions and is used as reference in terms of noise and coupling for the strain gauge implanted on it. Both gauges are incorporated into a half-Wheatstone bridge in order to cancel both, the noise and the electrical coupling by differential measurements. The released structure is actuated to out of plane vibrations by means of Lorentz forces on sinusoidal currents (in the gold path, c.f. figure 1) in a magnetic field provided by a permanent magnet. 
Preprint Measurement Science and Technology, Vol. 24, 2013, 084005 (9 pages) (doi:10.1088/09570233/24/8/084005)

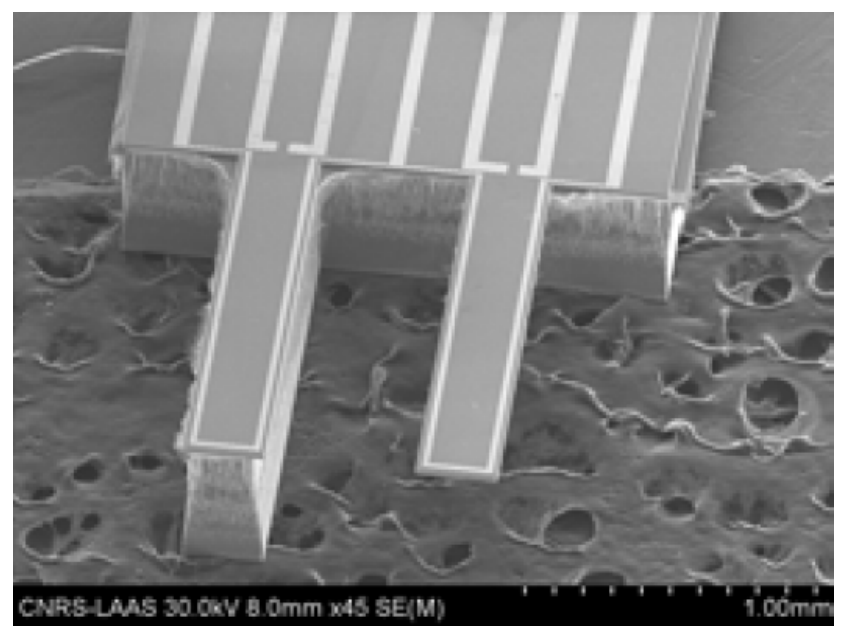

Figure 1. Example of microcantilevers fabricated at LAAS in Toulouse. Only the structure on the right is released

The second structure studied in this paper is named "U-shaped wire". The used wires are insulated nonmagnetic steel wires (the insulator is useful to avoid oxidation and corrosion problems). Their diameter is $79 \mu \mathrm{m}$ and their mass density is $7.5610^{3} \mathrm{~kg} \cdot \mathrm{m}^{-3}$. In previous studies, doubly clamped straight wires (or string) were investigated for characterizing liquids properties [4]. However, these doubly clamped beams may be subjected to instabilities in their resonant characteristics due to (e.g., thermally induced) varying normal stresses. In this study, a "U-shaped" wire, simply magnetically clamped on a support (as shown in figure 2) is used to overcome these spurious instabilities. The "Ushape" has been proven to be one of the best geometrical configurations to probe the physical properties of surrounding media $[8,9]$.
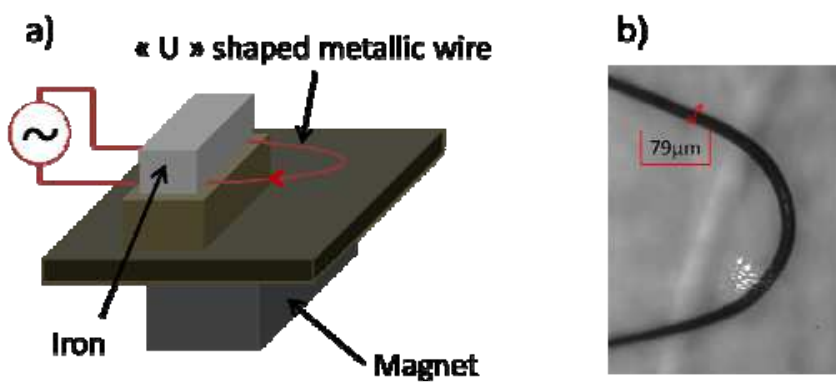

Figure 2. a) "U-shaped" wire actuating set-up;

b) Photograph of one of the examined wires immersed into a liquid.

These vibrating wires also use an electromagnetic actuation, as described above. In case of opaque fluids characterization, the steel wire has been clamped by soldering it onto a solid edge. This modification is necessary due to stability requirements and the lack of the ability visual control. In this case, the stability and the higher amplitude of the obtained electrical signal allow for accurate characterization of the mechanical resonance in opaque fluids.

\subsection{Measurements techniques}

Two methods have been used to characterize the mechanical resonance of both microstructures. For the first method, we used a laser vibrometer (MSA-500 Micro System Analyzer from Polytec). This technique is based on the interferences between a reference laser beam and another laser beam which 
is modulated by the structure's out-of-plane vibration. A picometer-range resolution is obtained when characterizing the investigated structures dynamic responses.

The second method utilizes a lock-in amplifier (Signal Recovery 7280 DSP) in order to measure the magnitude and phase of the motion-induced voltage in the electromechanical resonator. In the case of microcantilevers, the measured signal is on amplified Wheatstone bridge output voltage. For the wire, the input and the output signals (i.e. the actuation current and the motion induced voltage) are both carried by the conductive wire itself. Thus, the measured voltage consists of the ohmic voltage drop due to the wire's resistance and the motion-induced voltage. For both resonators, the first mechanical resonant mode has been measured electrically first in air and then in the examined liquids.

\subsection{Test fluids and process}

Three fluids have been characterized optically. Deionized water, a Newtonian fluid, was used for calibration. Then, two other complex viscous fluids have been investigated: hen egg albumin and a commercial shampoo (Garnier ${ }^{\circledR}$ FRUCTIS Shampoo) supposedly more viscous and exhibiting elastic properties. The measurements were performed in a cleanroom with temperature and humidity control. The temperature was varying between $18.8^{\circ} \mathrm{C}$ to $19.4^{\circ} \mathrm{C}$ and the humidity rate was $45 \%$.

Milk mixture turning into yogurt during fermentation as well as water have been characterized electrically, using the lock-in amplifier.

The mass density of the probed fluids has been measured at one temperature using a microbalance specific setup (using the Archimedes force principle) and the viscosity values have been investigated using our methods. The viscosity of pure water at $25^{\circ} \mathrm{C}$ is known to be $1 \mathrm{mPa} . \mathrm{s}(=1 \mathrm{cP})$ and is used as a reference value. These established data at $19{ }^{\circ} \mathrm{C}$ are summarized in Table 2.

Table 2. Density, viscosity and elasticity of probed fluids at $19^{\circ} \mathrm{C}$.

\begin{tabular}{lllll}
\hline Properties/Fluids & Water & Albumin & Shampoo & Yogurt \\
\hline Density (kg.m $\left.{ }^{-3}\right)$ & 998 & 1027 & 1018 & 1051 \\
Viscosity (mPa.s) & 1.03 & NA & NA & NA \\
Elasticity (Pa) & 0 & NA & NA & NA \\
\hline
\end{tabular}

NA : Not Available

\section{Modeling and treatment}

The differential equation describing the vibration of cantilevers (Euler-Bernoulli beam theory) is given as follows (this well-established equation is also presented e.g. in [10]):

$$
E I \frac{\partial^{4} w(x, \omega)}{\partial x^{4}}-m_{L} \omega^{2} w(x, \omega)=F_{\text {exc }}(x, \omega)+F_{\text {fluid }}(x, \omega)
$$

Here $w(x, \omega)$ is the amplitude of vibration at $x$ coordinate and at angular frequency $\omega$ in the $z$-direction $[\mathrm{m}], E$ is the Young's modulus [Pa], $I$ is the second moment of area of the cross section at coordinate $x$ 
with respect to the y axis $\left[\mathrm{m}^{4}\right], F_{\text {exc }}$ is the actuation force per unit length in x-direction $\left[\mathrm{N} . \mathrm{m}^{-1}\right] . F_{f l u i d}$ is the force per unit length in $x$-direction exerted by the fluid on the wire or cantilever [N.m $\left.{ }^{-1}\right], m_{L}$ is the mass per unit length of the microstructure $\left[\mathrm{kg} \cdot \mathrm{m}^{-1}\right]$. Equation (1) can be applied for both the cantilever and the U-shaped wire.

The hydrodynamic force per unit length exerted by the fluid on the microstructure can be written as:

$$
F_{\text {fluid }}(x, \omega)=-j \omega g_{1}(\omega) w(x, \omega)+\omega^{2} g_{2}(\omega) w(x, \omega)
$$

The cross section of the U-shaped vibrating is circular whereas it is rectangular in case of the microcantilever. This difference has an impact on the hydrodynamic force modeling, but a common formalism can be used for the terms $\mathrm{g}_{1}$ and $\mathrm{g}_{2}$ [11]:

$$
\begin{aligned}
& g_{1}=\pi \rho_{f} \frac{b^{2}}{4} \omega\left(b_{1} \frac{\delta}{b}+b_{2}\left(\frac{\delta}{b}\right)^{2}\right) \\
& g_{2}=\pi \rho_{f} \frac{b^{2}}{4} \omega\left(a_{1}+a_{2} \frac{\delta}{b}\right)
\end{aligned}
$$

with

$$
\delta=\sqrt{\frac{2 \eta}{\rho_{f} \omega}}
$$

where $b$ is the cantilever width or the wire diameter, $\eta$ is the fluid dynamic viscosity and $\rho_{f}$ is the mass density of the surrounding fluid. The coefficients $a_{1}, a_{2}, b_{1}$ and $b_{2}$ depend on the cross section geometry. For a circular cross section, $a_{1}=1, a_{2}=4, b_{1}=4, b_{2}=123 / 32$. This is obtained from a Taylor Series expansion when $\delta<<b$ of the expression given in [12]. For the rectangular cross section, these four coefficients have been determined by Maali et al.[11]. In our case they are, $a_{1}=1 \quad a_{2}=b_{1}=$ 3.7997 , and $b_{2}=2.7364$.

The complex shear modulus of the liquid $G$ is defined as:

$$
G=j \omega \eta
$$

It can be split up into the real (elastic) part $G^{\prime}$ and an imaginary (viscous) part $G^{\prime \prime}$. Thus, it follows that:

$$
\eta=-\frac{j G}{\omega}=-\frac{j\left(G^{\prime}-j G^{\prime \prime}\right)}{\omega}
$$

The transfer function of the mechanical resonator near the resonant frequency can be approximated by:

$$
H(L, f) \approx \frac{H_{0}}{1-\left(\frac{f}{f_{0}}\right)^{2}+2 \xi \frac{f}{f_{0}} j}
$$

Where $H_{0}$ is the static deflection, $f_{0}$ is the eigenfrequency and $\xi$ is the damping factor. The eigenfrequency $f_{0}$ is defined for a second order system as the free, undamped oscillation frequency. It is given by: 


$$
f_{0}=\frac{f_{0, \text { vacum }}}{\sqrt{1+\frac{g_{2}\left(f_{0}\right)}{m_{L}}}}
$$

Here $f_{0, \text { vacuum }}$ is the eigenfrequency in vacuum.

The damping coefficient $\xi$, which allows for estimating the decay of the transient oscillations is given by equation (10) which was already published in [13] and then used in [14]:

$$
\xi=\frac{g_{1}\left(f_{0}\right)}{4 \pi f_{0, \text { vacuum }} m_{L} \sqrt{1+\frac{g_{2}\left(f_{0}\right)}{m_{L}}}}
$$

Thus, if the fluid mass density is known, both, the real and imaginary parts of the shear modulus $G$ (previously developed in [15]) can be given by:

$$
\begin{gathered}
G^{\prime \prime}\left(f_{0}\right)=\frac{g_{1}}{D}-\frac{B}{D \sqrt{2 D}} \sqrt{\sqrt{\left(\frac{B^{2}}{D}+2\left(\omega g_{2}-C\right)\right)^{2}+4 g_{1}^{2}}-\frac{B^{2}}{D}-2\left(\omega g_{2}-C\right)} \\
G^{\prime}\left(f_{0}\right)=\frac{1}{D}\left(\omega g_{2}-C-\frac{B^{2} G^{\prime \prime}}{g_{1}-D G^{\prime \prime}}\right)
\end{gathered}
$$

Where:

$$
B=\frac{\pi b_{1}}{2 \sqrt{2}} b \sqrt{\rho_{f}} ; \quad C=\frac{\pi}{4} \rho_{f} b^{2} \omega ; \quad D=\frac{\pi b_{2}}{2 \omega}
$$

This model allows to calculate $G^{\prime}\left(f_{0}\right)$ and $G^{\prime \prime}\left(f_{0}\right)$ from $f_{0}$ and $\xi$.

The damping coefficient $\xi$ and the eigenfrequency $f_{0}$ are not extracted directly from the experimental spectra. Accuracy of the parameters $f_{0}$ and $\xi$ is a key point. An up-to-date method to obtain these parameters from an experimental spectrum has been developed in [16]. This method considers for second order system which are potentially affected by noise and electric coupling (cross talk). It allows subtracting noise and coupling for a significantly damped resonance. The method allows determing two parameters $f_{r}$ and $Q$ which are obtained with a great robustness eliminating the incertitude due to noise or coupling. The relation between these parameters and the damping coefficient and eigenfrequency are given here:

$$
\begin{aligned}
& \xi=\frac{1}{2 Q} \\
& f_{0}=f_{r}
\end{aligned}
$$

The presented model and parameter extraction are summarized in figure 3 .

This iterative method allows for fast determination of the complex viscosity or shear modulus. Furthermore, for calibration, only the eigenfrequency in vacuum is required, which is calculated from the measured value in air. This method has been applied to all of our (optical and electro-magnetical) measurements for both types of structures and for all of the investigated liquids. 
Preprint Measurement Science and Technology, Vol. 24, 2013, 084005 (9 pages) (doi:10.1088/09570233/24/8/084005)

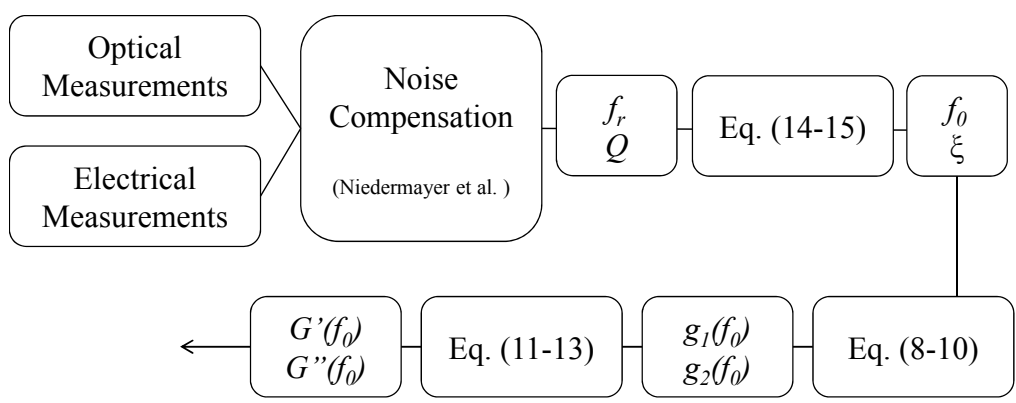

Figure 3. Synoptic of the presented model and parameter extraction.

In the case of cantilevers, an upgraded version of the presented model also exists. This version was initially presented by our team in [17] and improved in [18]. It allows determining not only $G^{\prime}\left(f_{0}\right)$ and $G^{\prime \prime}\left(f_{0}\right)$, but also $G^{\prime}(f)$ and $G^{\prime \prime}(f)$ over a rather large frequency range (half a decade) around the resonant frequency when the experimental data is only negligibly affected by noise and coupling; good results are obtained in the case of optical characterization. The upgraded version includes improvements which mainly concern the strong hypothesis made in equation (8) which is not outlined in the present paper. In section 4.1, the values for $G^{\prime}$ and $G^{\prime \prime}$ using cantilevers are obtained using this frequencydependent model.

\section{Experimental results}

\subsection{Optical characterizations (for transparent fluids)}

The measurements of the amplitude and phase of the displacement of the test structures have been performed using the three cantilever geometries and the U-shaped wire, in air and in the three test liquids using optical measurements. Sample measurements of the amplitude are presented in figures 4 and 5. As expected from theoretical considerations, these optical measurements show that an increase in viscosity results in increasing in damping ratio and a decrease in the resonant frequency. In the case of shampoo, optical measurements for cantilever LL and for the U-shaped wire do not show a resonant peak. Since these cases do not meet the above-mentioned modeling requirements, results will not be exploitable.

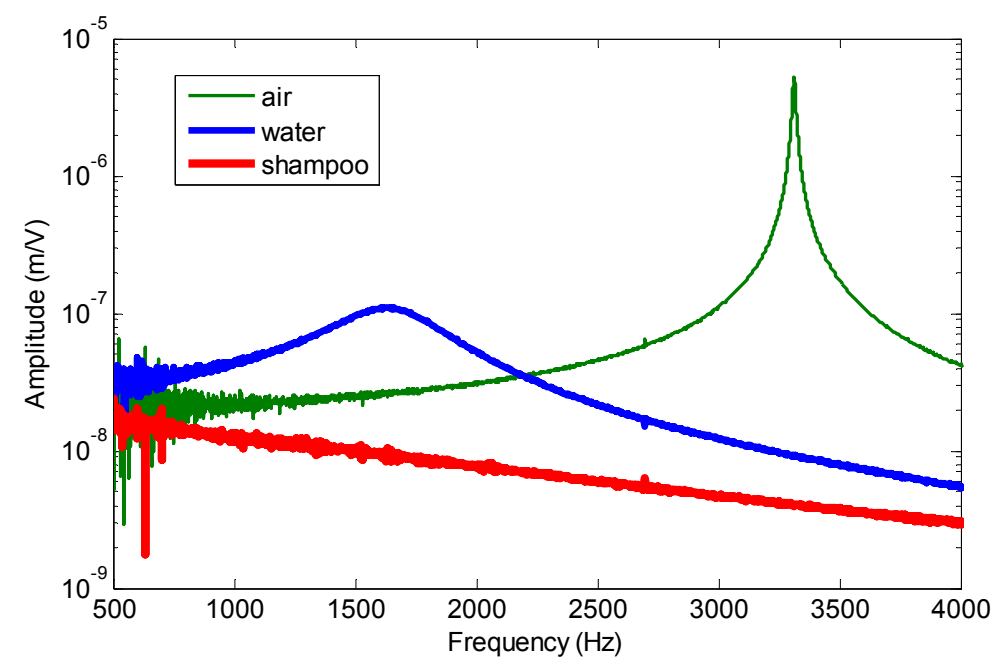

Figure 4. Cantilever LL free-end deflection spectrum obtained by optical vibrometry in air (green curve), water (blue), shampoo (red). 
Preprint Measurement Science and Technology, Vol. 24, 2013, 084005 (9 pages) (doi:10.1088/09570233/24/8/084005)

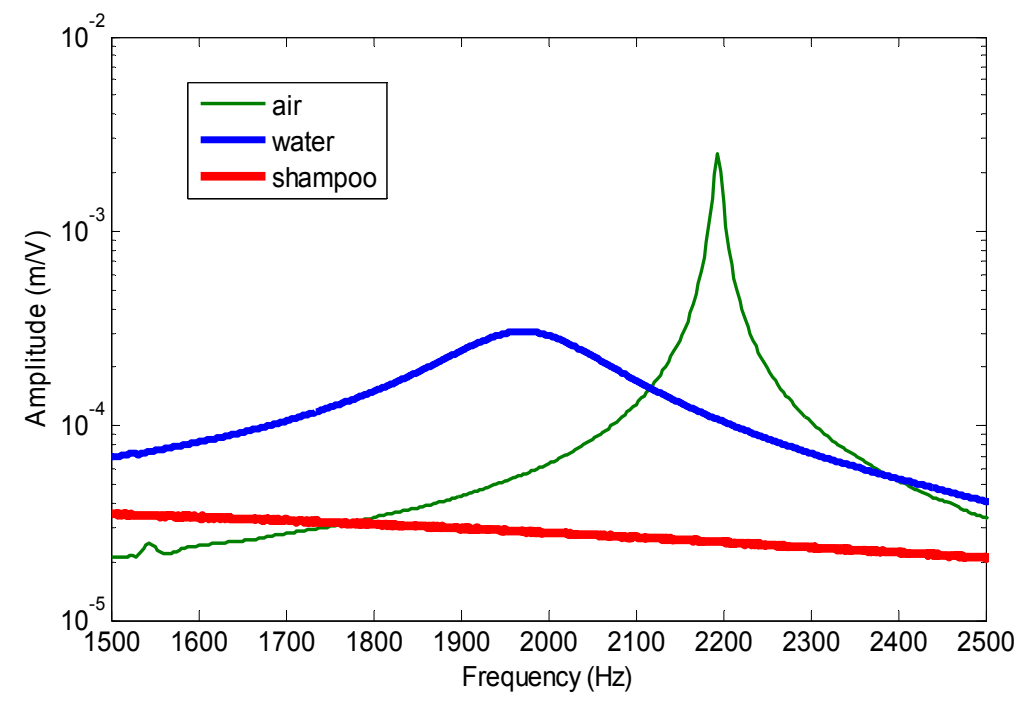

Figure 5. U-shaped wire deflection spectrum obtained by optical vibrometry in air (green curve), water (blue) and shampoo (red).

For both structures, the amplitude and phase data of the cantilever free-end deflection are directly provided by the laser vibrometer.

For the case of cantilevers it is possible to calculate the viscous modulus $G$ " at each frequency in a large range of frequencies around the resonant frequency. The resulting rheograms are presented in Figure 6 for the three test liquids.

For U-shaped wires, the damping ratio $\xi$, the eigenfrequency in liquid $f_{0}$, and the eigenfrequency in air (which is approximately the same as in vacuum, $f_{0 \text {,vacuum }}$ ) have been extracted from the spectrum obtained by vibrometry (when a resonant peak have been observed). The presented model has been used to calculate the viscous modulus at the eigenfrequency $\left(G^{\prime \prime}\left(f_{0}\right)\right)$ in the three examined liquids. In figure 6 , the single values $G$ "' $\left(f_{0}\right)$ obtained with U-shaped wires and the ranges of values $G$ " $(f)$ obtained with cantilevers A, LL and LH in the three different liquids are presented. 

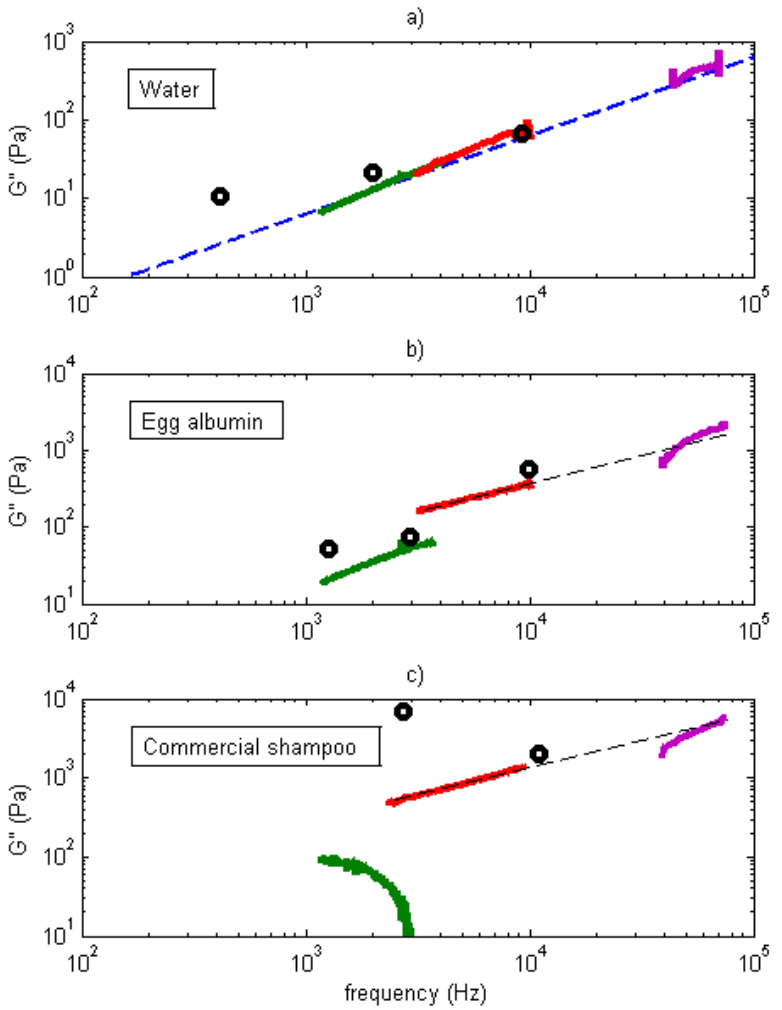

Figure 6. Rheograms: viscous part $G$ " of the shear modulus calculated for cantilevers LL (green), LH (red), A (purple) and the U-shaped wires (o) in a) deionized water, b) hen egg albumin and c) a commercial shampoo. The theoretical viscous modulus of water is also depicted (--blue).

The behavior of the viscous modulus of water is well known (blue dotted curve in figure 6.a). The results obtained with cantilevers have been used to calibrate modeling parameters to obtain the Newtonian behavior of this reference fluid. The measurements performed with U-shaped wires only require calibrating $f_{0, a i r}$. For non-Newtonian fluids, the modeling parameters calibrated in water have been used. In both cases, for egg albumin and shampoo, the rheograms of cantilever LH and A follow the same trends (represented by the dashed lines in figure 6.b) and c)). The recorded spectra with cantilever LL did not exhibit a resonant behavior in the case of shampoo, and exhibited a strong damping in the case of egg albumin. In these cases, as noise and coupling are relatively large in respect to the low signal the upgraded model cannot be applied. Therefore, the viscous modulus corresponding to cantilever LL is inconsistent.

The values of $G$ " calculated using the U-shaped wires are less accurate but they remain of the same order of magnitude without any calibration step. They were obtained with a magnetically clamped wire for which the accuracy of the final measurement is affected by stability issues as mentioned in section 2.1. More stability, reproducibility and accuracy on the viscosity measurement are achieved and detailed in the section 4.1 using a soldered clamping for the U-shaped structure.

In order to validate the calculating method detailed in this paper, the optical characterization of a microcantilever of geometry LH (see Table 1) has been performed in deionized water from $19^{\circ} \mathrm{C}$ to $50{ }^{\circ} \mathrm{C}$ (using a Peltier element for temperature control). The measured magnitudes are shown in figure 7.a and the dependence of the quality factor with respect to temperature in figure 7.b. The viscosities 
have been calculated using the presented model, see figure 7.c, considering the module of the complex viscosity (see equation 7).
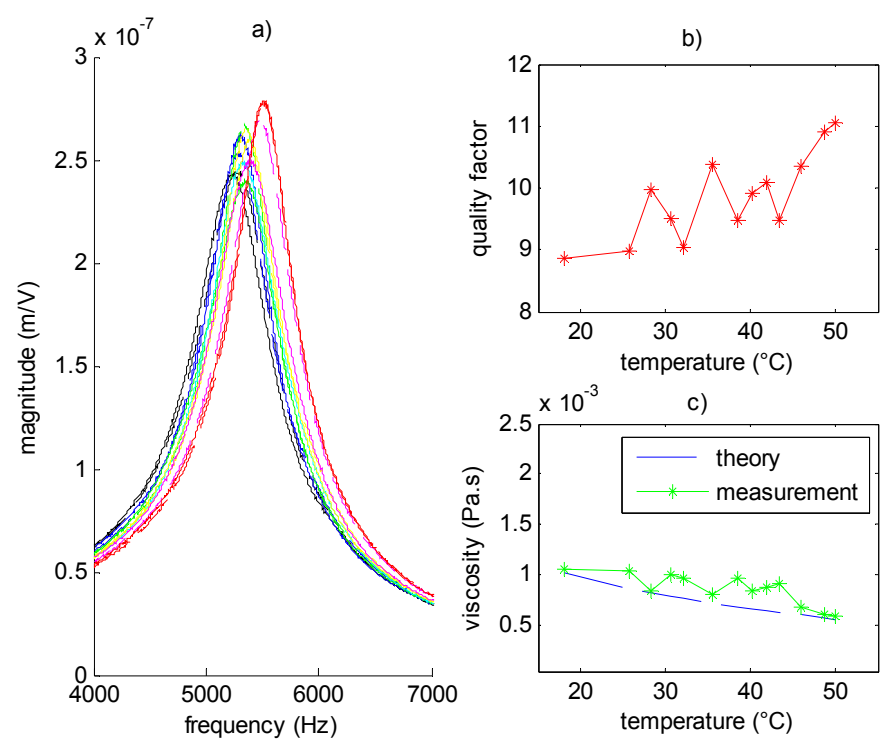

Figure 7. a) Magnitudes measured optically with cantilever LH immersed into water from $19{ }^{\circ} \mathrm{C}$ (dark line) to $50^{\circ} \mathrm{C}$ (red line). b) Quality factor with respect to temperature. c) Module of the complex viscosity calculated using the presented model (the theoretical trend is represented by the blue dashed line).

As expected, the quality factor of the LH cantilever increases with temperature due to the decrease of the viscosity of water. The viscosity of water measured between $19{ }^{\circ} \mathrm{C}$ and $50^{\circ} \mathrm{C}$ follows the expected trend of theoretical dependence of the water's viscosity with respect to temperature (dashed blue line in figure 7.c). The great accuracy obtained with the optical measurements combined with the presented method allows calculating accurately the viscosity of water without any calibration. The same kind of measurements has to be tested for opaque viscoelastic liquids to confirm and validate the potential of the method.

\subsection{Electrical characterizations (transparent and opaque liquids)}

\subsubsection{Preliminary results}

Electrical measurements are performed for both structures using a lock-in amplifier, as mentioned in section 2.2. Some results are shown in figures 8 and 9 .

For the case of cantilevers, the influence of the fluid on the signal, even if clearly observable, could not be determined due to a dominant electrical coupling. In air, a first approach of the electrical coupling can be modeled by a simple constant capacitive circuit and the cantilever resonance clearly overshoots the coupling base line (cf. figure 8). However, the modeling of coupling is much more complex in liquids. There, the coupling is affected by the surrounding liquid properties (e.g., electrical conductivity). Concretely, this means that the results from figure 8 are not reproducible. More generally speaking, in aqueous media, different cantilever spectrum acquisitions at different times give different results because of the variation of electrical coupling. This instability is a real limitation for continuous non-optical acquisition with respect to time. The reproducibility of electrical measurements 
Preprint Measurement Science and Technology, Vol. 24, 2013, 084005 (9 pages) (doi:10.1088/09570233/24/8/084005)

of cantilevers in water-based solution has still to be improved in order to measure continuously an exploitable signal for viscosity monitoring measurements.

In the case of U-shaped wires, measurements in air and in water show more stability and reproducibility. As shown in figure 9, a reproducible and almost noiseless signal is obtained with the wire in both cases. The amplitude's resonance peak dynamic is the principal remaining problem: the motion-induced voltage is small compared to the driving voltage which can consume the main part of the digitalization scale. The numeric resolution has to be forced to the order of $\mathrm{nV}$ to be able to obtain a sufficient resolution for rheology purposes. A dedicated finely set differential compensation circuitry could solve this problem for future acquisitions (being careful about not restoring coupling issues).

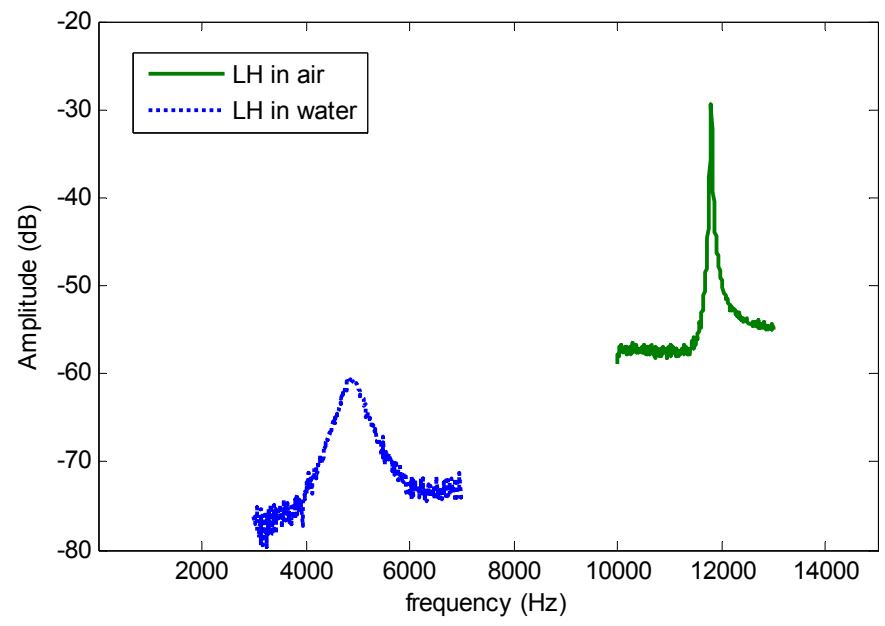

Figure 8. Amplitudes containing the fundamental resonant frequency measured piezoresistively for cantilever LH in air (green line) and in water (dotted blue)

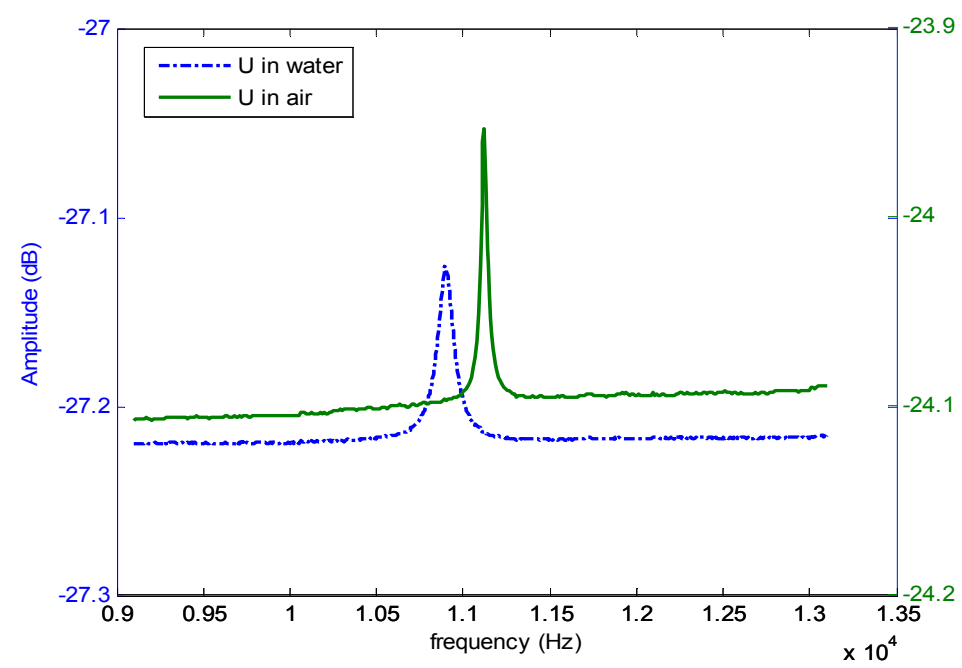

Figure 9. Amplitudes of the motion induced voltage containing the fundamental resonant frequency for a U-shaped structure in air (green line) and in water (dotted blue line) 
Preprint Measurement Science and Technology, Vol. 24, 2013, 084005 (9 pages) (doi:10.1088/09570233/24/8/084005)

\subsubsection{Viscosity monitoring experiment with U-shaped wires}

A viscosity monitoring experiment has been performed in order to completely validate the proposed method in the case of an opaque complex liquid mixture. For this purpose, the viscosity of yogurt was examined during fermentation. The experiment has been successfully reproduced three times using Ushaped structures. Some photographs and raw data of one experiment are presented in figure 10 . The measurements have been done using an U-shaped structure packaged with a magnet. The U-shaped sensor has been immersed together with thermocouples (for temperature control) in the prepared milkmixture.

First, $168 \mathrm{~mL}$ of full-cream milk is mixed with $32 \mathrm{~mL}$ of yogurt. Then a first mass-density measurement was performed with a microbalance specific set-up; at $9{ }^{\circ} \mathrm{C}$ it was $1035.5 \mathrm{~kg} . \mathrm{m}^{-3}$. The mass-density was measured 15 hours later again, at $19.2^{\circ} \mathrm{C}$, after the milk has turned into yogurt (see figure 10.c). At $19.2^{\circ} \mathrm{C}$, it was $1051.4 \mathrm{~kg} \cdot \mathrm{m}^{-3}$ (this last measurement is more complicated: due to the specific viscoelastic properties of yogurt, the measurement has to be stabilized during 2 hours to obtain a consistent value). In between, the mixture was heated to a temperature close to $37^{\circ} \mathrm{C}$ during 8 hours. The fermentation process occurs during this period. The deflection spectrum of the U-shaped structure used for this experiment has been measured in air before (cf. figure 10.c). The amplitude and phase of the motion induced voltage are continuously acquired by the lock-in amplifier. Both, the resonant frequency and the quality factor are calculated in real time after each spectrum acquisition using the correction developed in [16]. The milk-mixture is simply heated by a hot plate and temperature is measured every minute during the experiment using the thermocouples. Once the temperature of the mixture is close to $37^{\circ} \mathrm{C}$ the fermentation process starts efficiently and after 100 minutes the quality factor starts to decrease (cf. figure 10.d). The mixture becomes more viscous. Once the yogurt is formed, the resonant frequency starts to decrease a lot (see figure 10.b).
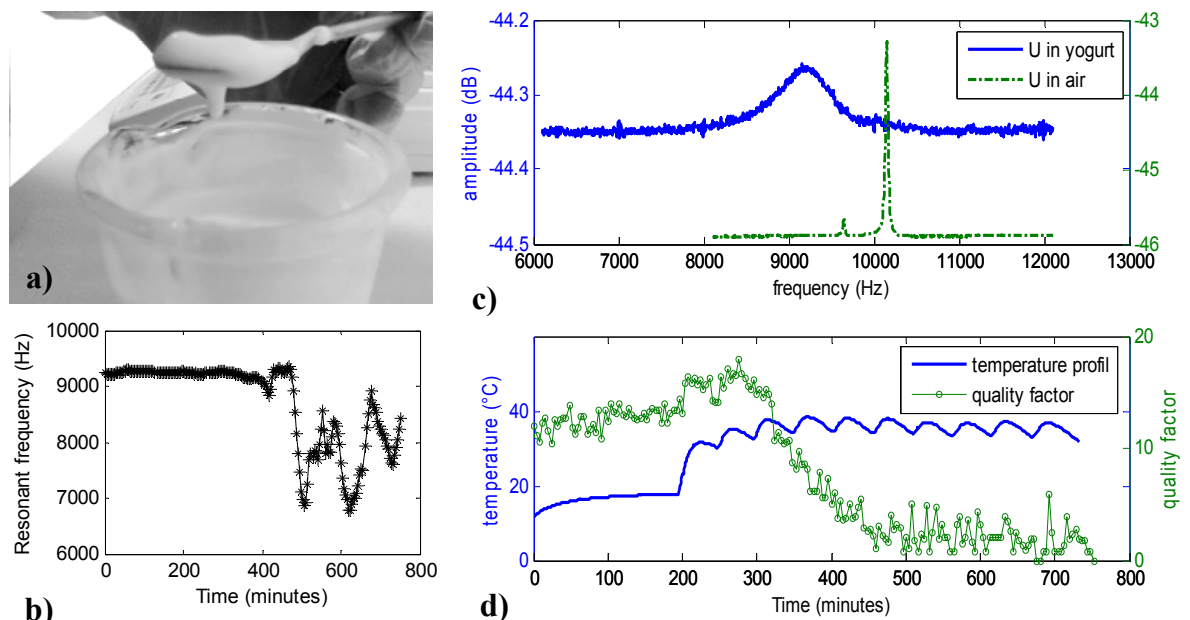

Figure 10. a) Resultant yogurt. b) Measured resonant frequency with respect to time. c) Measured signal in air and yogurt. d) Temperature profile applied during the fermentation process (blue line), and measured quality factor (dotted green).

The elastic modulus, the viscous modulus and the complex viscosity have been calculated using the presented model. The measured mass density is used in the calculation. The complete monitoring of yogurt viscosity during the fermentation process is achieved and results are presented in figure 11. In this experiment, the viscosity is measured by the damping of a mechanical resonator and the associated resonant frequency shift.

In literature, the most widely used method for yogurt viscosity monitoring is performed by measuring the acidity of the mixture and calculating the viscosity with respect to the $\mathrm{pH}$ value [6]. Viscosity 
Preprint Measurement Science and Technology, Vol. 24, 2013, 084005 (9 pages) (doi:10.1088/09570233/24/8/084005)

versus time curves is obtained, comparable to the one produced in figure 11.a. In [6], authors have a starting plateau around $10 \mathrm{mPa}$.s and a final plateau around $200 \mathrm{mPa}$.s. Here, the starting plateau is around $4 \mathrm{mPa}$.s and the final one around $190 \mathrm{mPa}$.s. In between, results slightly differ: the dynamics of fermentation are not the same, due to different temperature profiles (up to $80^{\circ} \mathrm{C}$ in [6]) and of course due to the use of different types of ferments. Thus, the values of viscosity measured in this experiment are consistent and valid, close to literature's reference data (see discussion below).

Furthermore, it is stated in literature, that the viscoelasticity of yogurt can be continuously measured with a temperature controlled rheometer. The elastic and the viscous modulus may be measured in oscillation mode at $1 \mathrm{~Hz}$ [7], and compared to the rheograms presented in figure 11.b.
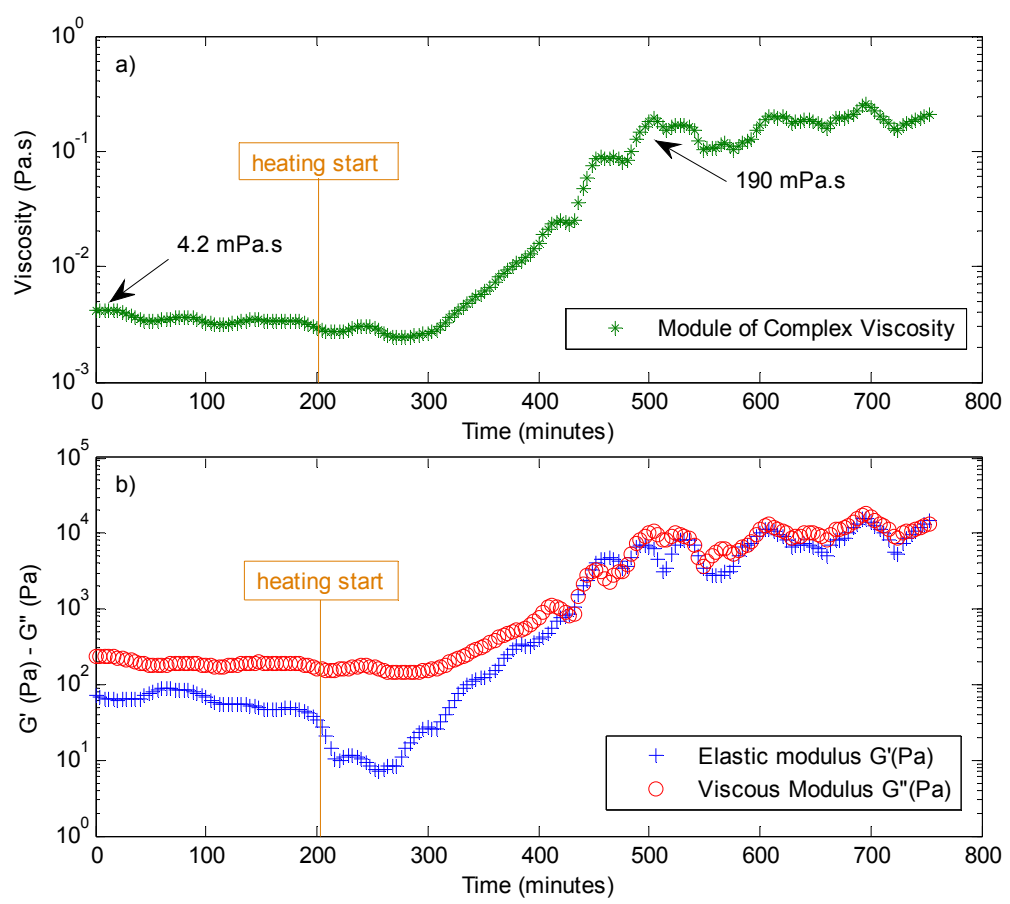

Figure 11. Viscosity monitoring during yogurt fermentation process a) Module of complex viscosity (Pa.s) with respect to time (minutes). b) Elastic (G') and viscous modulus (G') in Pa with respect to time.

After the 500 minutes, $G^{\prime}$ and $G$ " have the same order of magnitude, which means that the viscoelastic character of yogurt is obtained. A quantitative comparison between results from literature [7,19] and the results presented in figure 11.b is not possible due to the differences of operating frequencies ( 1 to $200 \mathrm{~Hz}$ using rheometers and $9.2 \mathrm{kHz}$ using this sensor). However, the trends are remarkably comparable and, in both cases, the ratio of $G^{\prime}$ (after fermentation) and $G^{\prime}$ (before fermentation) are similar, very close to 200 . The same verification was successfully achieved with the $G$ ” modulus. The presented rheograms are consistent in terms of variation of moduli.

The comparison of this investigation with literature shows that, the performed viscosity monitoring process during yogurt fermentation is valid.

\section{Discussion}

The current state-of-the-art high-frequency rheology shows deficits in terms of reproducibility of the measured values. Rheologists state that a margin of $20 \%$ of difference between supposedly 
Preprint Measurement Science and Technology, Vol. 24, 2013, 084005 (9 pages) (doi:10.1088/09570233/24/8/084005)

identical experimental values obtained by different teams is acceptable [20]. The presented model is sufficient to accurately measure a valid complex viscosity compared to literature's data.

Due to their geometry, U-shaped structures allow minimizing the mechanical losses (as compared to cantilevers) which is an important issue when performing measurement in viscous media. Moreover, the electrical detection was sufficiently free of noise or electrical coupling for measuring accurately the desired resonance parameters $\left(f_{r}\right.$ and $\left.Q\right)$ and therefore, for performing viscosity monitoring in significantly viscous opaque liquids. Nevertheless this method needs a resonant peak to extract $f_{r}$ and $Q$; this is a limitation which not allows to characterize fluids of high viscosity.

Compared to U-shaped structures, the microcantilevers used in this experiment were not suitable for opaque fluid characterization, mainly because of both, the electrical coupling and the mechanical damping. However, in the case of an accurate optical characterization, a good stability to characterize liquids of relatively small viscosity is demonstrated. Furthermore, an accurate frequency dependent model is available and allows calculating rheological parameters not only at the resonant frequency but also over a range of frequency around this value [18]. Thus, the accuracy of this frequency dependent method remains insufficient in case of strongly damped spectra (cf. figure 6, 'commercial shampoo') due to some modeling limitations and to an excessive sensitivity to the existing incertitude about some parameters.

The detailed experiments associated to the proposed modeling have shown a great potential for viscosity monitoring of various viscoelastic products. The obtained results qualify the U-shaped sensor for viscosity monitoring. Improvements can be performed in order to reach better performances especially in term of quality factor. The clamping and the stiffness can be improved. The silicon cantilever has to be strongly modified in order to measure an electrical signal in opaque liquids with less electrical coupling. A U-shaped silicon based structure could be an option to demonstrate better performances and an increased integration. Nonetheless, the actual U-shaped structure is low-cost and low energy consuming; the change of both, the material and the clamping technique could be sufficient to obtain satisfying performances. Thus, a future sensor could cover a viscosity range from 0.5 to $1000 \mathrm{mPa}$.s. The actual system allows for measuring viscosities up to $250 \mathrm{mPa}$.s.

\section{Conclusion}

As a conclusion, microcantilevers and vibrating wires are both potential viscosity sensors. The actual U-shaped design allows minimizing the damping losses and the electrical coupling; furthermore, it features easy fabrication and handling as well as low cost production. Results obtained with this structure allow considering it as a real microrheometer which could be integrated in an array of such structures. The simplicity of this design is its main asset, since a simple geometry can be modeled easily and because the model itself drives an important part of the accuracy of the viscoelasticity estimation. On the other hand, manufacturing of microcantilevers is more expensive; however a higher sensitivity can be achieved when using optical detection in a low viscous liquids. The validity and the accuracy of the viscosity calculated without calibration using the U-shaped structure is acceptable, reliable and integrated (i.e. no optical measurements required). The geometry associated with this robust analytical method appears really promising for future application.

The accuracy of both measurement methods can still be improved. Thus, the presented results with these simple micro-mechanical-structures have proven their applicability as micro-sensors performing microrheological measurements in a frequency range which has hardly been investigated so far. Finally the integrated viscosity monitoring is now available using this method for many kinds of viscoelastic liquids. 


\section{Acknowledgments}

The authors would like to thank Cédric Ayéla for the cantilevers mask design, Liviu Nicu, Fabrice Mathieu and Daisuke Saya for the fabrication of the silicon cantilever, Alexander Niedermayer for the resonant frequency and quality factor extraction procedure. This work was partially supported by the French National Agency (MicRheo project $\mathrm{n}^{\circ}$ ANR-08-NANO-004) and by the Conseil Régional d'Aquitaine $\left(\mathrm{n}^{\circ} 20091102001\right)$. The contributions of Martin Heinisch and Bernhard Jakoby were supported by the Austrian Competence Center in Mechatronics (ACCM). The collaboration between Austria and France has been founded by a project PHC-AMADÉUS.

\section{References}

[1] S.J. Martin, V.E. Granstaff, G.C. Frye, Characterization of a quartz crystal microbalance with simultaneous mass and liquid loading 1981 Anal. Chem. 63 pp.2272-2281

[2] B. Jakoby, R. Beigelbeck, F. Keplinger, F. Lucklum, A. Niedermayer, E. Reichel, C. Riesch, T. Voglhuber-Brunnmaier, B. Weiss, Miniaturized sensors for the viscosity and density of liquids performance and issues 2010 IEEE Trans. on Ultrason., Ferroelec. and Freq. Contr. 57 pp.111-120.

[3] I. Dufour, A. Maali, Y. Amarouchene, C. Ayela, B. Caillard, A. Darwiche, M. Guirardel, H. Kellay, E. Lemaire, F. Mathieu, C. Pellet, D. Saya, M. Youssry, L. Nicu, A. Colin, The Microcantilever: A Versatile Tool for Measuring the Rheological Properties of Complex Fluids 2012 Journal of Sensors, Volume 2012 Article ID 719898

[4] M. Heinisch, E.K. Reichel, I. Dufour, B. Jakoby, Tunable resonators in the low kHz range for viscosity sensing 2012 Sensors and Actuators A, Vol 186, pp. 111-117.

[5] J. E. Sader, Frequency response of cantilever beams immersed in viscous fluids with applications to the atomic force microscope 1998 J. Appl. Phys. 84 pp.64-76

[6] C. Soukoulis, P. Panagiotidis, R. Koureli, C. Tzia, Industrial Yogurt Manufacture: Monitoring of Fermentation Process and Improvement of Final Product Quality 2007 Journal of Dairy Science Volume 90 Issue 6 Pages 2641-2654

[7] W. J. Lee, J. A. Lucey, Formation and Physical Properties of Yogurt 2010 Asian-Aust. J. Anim. Sci. Vol. 23, No. 9 : 1127 - 1136

[8] A. Agoston, F. Keplinger, B. Jakoby , Evaluation of a vibrating micromachined cantilever sensor for measuring the viscosity of complex organic liquids 2005 Sensors and Actuators A: Physical 123124 , pp. 82-86.

[9] F. Keplinger, R. Beigelbeck, F. Kohl, S. Kvasnica, A. Jachimowicz, B. Jakoby, Frequency and transient analysis of micromachined U-shaped cantilever devices for magnetic field measurement 2005 The 13th International Conference on Solid-State Sensors TRANSDUCERS '05 vol.1, no., pp. 630- 635 Vol. 1.

[10] A. H. Nayfeh, P. F. Pai, Linear and Nonlinear Structural Mechanics 2008 John Wiley \& Sons 
Preprint Measurement Science and Technology, Vol. 24, 2013, 084005 (9 pages) (doi:10.1088/09570233/24/8/084005)

[11] A. Maali, C. Hurth, R. Boisgard, C. Jai, T. Cohen-Bouhacina and J.P. Aimé, Hydrodynamics of oscillating atomic force microscopy cantilevers in viscous fluids 2005 J. Appl. Phys. 97, 074907, 6 pages

[12] L. Rosenhead, Laminar Boundary Layers $\sim$ Clarendon, Oxford, 1963.

[13] M. Youssry, N. Belmiloud, B. Caillard, C. Ayela, C. Pellet, I. Dufour, A straightforward determination of fluid viscosity and density using microcantilevers: from experimental data to analytical expressions", 2011 Sensors and Actuators A, vol. 172, pp. 40-46.

[14] M. L. Mather, M. Rides, C. R. G. Allen, Paul E. Tomlins, Liquid viscoelasticity probed by a mesoscale piezoelectric bimorph cantilever 2012 J. Rheol. 56, 99, 14 pages.

[15] N. Belmiloud, I. Dufour, L. Nicu, A. Colin, J. Pistré, Vibrating microcantilever used as viscosimeter and microrheometer 2006 IEEE Sensors conf., Daegu, Korea, pp. 753-756.

[16] A O Niedermayer, T Voglhuber-Brunnmaier, J Sell, B Jakoby, Methods for the robust measurement of the resonant frequency and quality factor of significantly damped resonating devices 2012 Meas. Sci. Technol. 23085107

[17] N. Belmiloud, I. Dufour, A. Colin, L. Nicu, Rheological behavior probed by vibrating microcantilevers 2008 Applied Physics Letters 92, 041907

[18] M. Youssry, E. Lemaire, B. Caillard, A. Colin, I. Dufour, On-chip characterization of the viscoelasticity of complex fluids using microcantilevers, 2012, Measurement Science and Technology, Vol. 23125306 (10pp).

[19] A. N. Hassan, R. Ipsen, T. Janzen, K. B. Qvist, Microstructure and Rheology of Yogurt Made with Cultures Differing Only in Their Ability to Produce Exopolysaccharides 2003 J. Dairy Sci. $86: 1632-1638$

[20] M.Buchanan, M. Atakhorrami, J. F. Palierne, F. C. MacKintosh, C. F. Schmidt, High-frequency microrheology of wormlike micelles 2005 Physical Review E 72, 011504. 